Article

\title{
Nanoscale Silicon Waveguide Based Thermo-Optic Sensor Using a Compact Mach-Zehnder Interferometer
}

\author{
Conrad Rizal1,2* and Boris Niraula ${ }^{2}$ \\ ${ }^{1}$ Department of Physics, Baylor University, 1301 S University Parks Dr, Waco, TX, 76798, USA \\ ${ }^{2}$ Department of Electrical and Computer Engineering, University of British Columbia, Vancouver, BC, \\ Canada. \\ *Correspondence: Conrad Rizal@baylor.edu , Tel.: +1-254-218-9096
}

\begin{abstract}
A compact Mach-zehnder interferometer with a novel design of directional couplers and a phase shifter has been presented as a thermo-optical sensor. With the aim of reducing device size to micro and nano dimension silicon-on-insulator technology was employed. That allowed miniaturization of device size through the reduction of its cross sectional area to $0.066 \mu \mathrm{m}^{2}$ and the radius of curvature of both the arms of the directional coupler and S-bends of the phase shifter to $5 \mu \mathrm{m}$ and C-bends to $3 \mu \mathrm{m}$. These nano size device dimensions made it possible to reduce the coupling gap to $0.2 \mu \mathrm{m}$, which resulted in a significant reduction in the coupling length. The device geometry and its performance characteristics were analyzed and optimized using coupled mode analysis and finite difference time domain simulation tools, respectively. The wavelength dependent transmission loss of the device was measured at different temperature to verify and validate its performance characteristics. Tested devices showed a remarkable temperature dependent transmission characteristic offering significant changes in transmission loss band - with as low as $0.45{ }^{\circ} \mathrm{C}$ change in substrate temperature. The extinction ratio and the free spectral range of the device were $26 \mathrm{~dB}$ and $0.26 \mathrm{~nm}$ respectively in the wavelength range of $1549.5 \mathrm{~nm}-1550.5 \mathrm{~nm}$. These results imply that the devices presented here can be used as compact and highly sensitive thermal sensors and optical switches.
\end{abstract}

Keywords-Nanoscale silicon, optical waveguides, Mach-zehnder interferometer, directional coupler, thermal sensor, optical switches

PACS: 42.79.Ta, 42.79.Qx, 42.79.Gn, 42.82.-m, 42.82.Et, 78.20.B, 78.20.Nv

\section{Introduction}

Silicon based Mach-zehnder interferometers (MZI) are used for optical switching and sensing applications for the past few decades, as they show large thermo-optic effect, high optical index difference, low propagation and coupling losses, and the availability of state-of-the-art silicon on insulator (SOI) technology for their fabrication [1-13]. 
Two practical considerations make these MZI attractive as thermo-optical sensors: the possibility of changing the wavelength dependent refractive index through small change in temperature and the remarkable sensitivity of their transmission band / spectra to a small change in externally applied stimuli such as electric field and temperature as silicon possesses high thermo-optical coefficient of $1.82 \times 10^{-4} \mathrm{~K}^{-1}$ and high thermal conductivity of $149 \mathrm{~W} \cdot \mathrm{m}^{-1} \cdot \mathrm{K}^{-1}$ [2-6,12,14-37].

Most MZI devices based on conventional materials suffer from large bending losses because of large device size, as it is difficult to make the arms of the directional coupler (DC) and C-bends of the phase shifter with smaller radius of curvature using conventional technology [5,15,32]. The devices made using conventional technologies are usually in the range of hundreds of micrometers to centimeters leading to large gap between coupling waveguides, a significantly long coupling length, and a large dimension of the phase shifter arm [38,39].

SOI based technology not only offers the possibility of reducing device dimension significantly, but it also allows a significant reduction of power loss through reduction of bending radius of both the sigmoidal arm of the DC coupler and C-bends of the phase shifter. For example, Si waveguides fabricated using SOI technology offers the opportunity of reducing device dimensions by one order of magnitude and the bending radius of the sigmoidal (S) arm to $5 \mu \mathrm{m}$ and C-bends to $3 \mu \mathrm{m}$, as opposed to devices that are several orders of magnitude larger when fabricated using conventional semiconductor technology [4,40-42].

Various nanoscale Si based waveguides have been reported in literature earlier [24-29]. However, only a few reports have been published on highly compact optical MZI-based thermal sensors and switches, with waveguide cross-sectional area of $0.066 \mu \mathrm{m}^{2}$, radius of curvature of S- and C-bends with $5 \mu \mathrm{m}$ and $3 \mu \mathrm{m}$, respectively, and strong thermo-optic effect at as low as $0.45^{\circ} \mathrm{C}$ change in substrate temperature $[5,19,41,43]$. Besides reporting optical characteristics of the MZI device, consisting of DC couplers and phase delay arms, using transfer matrix method, we report optical signal propagation mode for these devices using finite difference time domain (FDTD) and Matlab Mode solver. This has been best achieved by dividing the entire device module into 3 parts: DC splitter, phase delay arms, and DC combiner.

Optimization of geometrical parameters of the device and its optical characteristics were performed using coupled mode analysis. Finite element method and FDTD tools were employed to analyze and validate the profile of the electromagnetic properties of the device. Utilizing the advantage of nano- scale cross sectional area of the Si waveguide and small radius of curvature of its DC coupler and C-bends of the phase shifter arms, we demonstrate a novel compact thermo-optical sensor based on MZI configuration using SOI technology. The following aspects of the device are 
also elaborated in detail: mask-layout, verification of design concept, fabrication protocol, device testing protocol, measurement of optical properties, and verification of test results.

\section{Principle and Design}

The performance characteristics of a compact MZI-based optical device strongly depends on the optimization of device configuration, design parameters and device sizes - including on the dimension of the following components: directional coupler (DC), sigmoidal sensing arms, C-bends, and heating element. We started with modeling and optimization of geometric parameters of the DC coupler followed by straight and Sigmoidal arms of the MZI and heating element.

Three important parameters that determine the performance characteristics of the DC are coupling length, $l_{c}(\lambda)$, gap between two straight arms of the couplers (g), and coupling coefficient $(\kappa)$. In a DC waveguide configuration reported in this paper, where $g$ is in nanometer range, the input optical signal to the waveguide couples with the optical signal from a second waveguide, as a result of overlapping of the evanescent field of each other. The $\kappa$ value in turn depends on waveguide parameters such as wavelength $(\lambda)$ of the input light and g. The DC is also characterized in terms of difference in propagation constant $(\Delta \beta)$, and the interaction length of the waveguide, and effective refractive index $\left(n_{\mathrm{eff}}\right)$, where $\Delta \beta$ is related to $n_{\mathrm{eff}}$ and $\lambda$ as, $\Delta \beta=2 . \pi \cdot\left(\frac{n_{e f f 2-} n_{e f f 1}}{\lambda}\right)$, where, $n_{e f f 1}$ and $n_{e f f 2}$ are the effective refractive indices of wave guide 1 and 2, respectively. The operation of this type of DC with identical wave-guiding arms can be described by the coupled mode equations as: $P_{3(\lambda)}=\cos ^{2}[\kappa \pi]$ and $P_{4(\lambda)}=\sin ^{2}[\kappa \pi]$, where, $\kappa$ is an exponentially decaying function and it varies with $g$ and $P_{3(\lambda)}$ and $P_{4(\lambda)}$ are $\lambda$ dependent output powers. The $l_{c}(\lambda)$ can be determined using the relationship as: $l_{c(\lambda)}=\frac{\pi}{4 l_{c}(\lambda)}$. The $l_{c}(\lambda)$ is further related to effective change of refractive index $\left(\Delta n(\lambda)_{e f f}\right)$ as: $l_{c(\lambda)}=\frac{\pi}{4 \Delta n(\lambda)_{e f f}}$, where, $\Delta n(\lambda)_{e f f}$ is the difference in the symmetric and anti-symmetric effective mode indices. Note that this relationship must be satisfied in order to achieve splitting of input power between the two arms of the couplers in equal amount.

As shown in Figure 1(a), the transfer matrix for the DC splitter is the product of power transmission co-efficient $\left(\gamma_{\mathrm{s}}\right)$ and mode coupling factors $\left(p_{i}, q_{i}\right)$ as: $M_{s}=\gamma_{\mathrm{s}}\left(\begin{array}{cc}p_{1} & j \cdot q_{1} \\ j \cdot q_{1} & p_{1}\end{array}\right)$, where, $p_{1}=\sqrt{1-\kappa_{s}}, q_{1}=-j \sqrt{\kappa_{s}}, \kappa_{s}$ is the coupling coefficient of the splitter, $\gamma_{\mathrm{s}}$ is the transmission coefficient of the splitter, and $p_{1}^{2}+q_{1}^{2}=1$. Similarly, the transfer matrix for the DC combiner can be given by: $M_{c}=\gamma_{c}\left(\begin{array}{cc}p_{2} & j \cdot q_{2} \\ j \cdot q_{2} & p_{2}\end{array}\right)$, where, $p_{2}=\sqrt{1-\kappa_{c}}, q_{2}=-j \sqrt{\kappa_{c}}$. Note that $\kappa_{c}$ is the coupling coefficient of the combiner, $\gamma_{c}$ is the transmission co-efficient of the combiner, and $p_{2}^{2}+q_{2}^{2}=1$. For a lossless operation, the values of $\gamma_{s}$ and $\gamma_{c}$ are equal to one each and $\kappa_{s}$ and $\kappa_{c}$ equal to $1 / 2$ each provided the power gets split and combined equally in each DC. As shown in Figure 1(b), the device 
is divided into 3 parts so as to accurately calculate its optical properties: splitter, phase delay arm, and combiner.

The device can also be modeled as a cascade of two 2-ports. The splitter and combiners, which are DCs, in turn consist of two ports each. $P_{1}^{\text {in }}, P_{2}^{\text {in }}$ denote the input powers to the device, whereas $P_{1}^{\text {out }}, P_{2}^{\text {out }}$ denote the output powers from the device corresponding to $a_{1}^{\text {in }}, a_{2}^{\text {in }}$ and $a_{1}^{\text {out }}, a_{2}^{\text {out }}$, respectively, where $a_{1}^{\text {in }}, a_{2}^{\text {in }}$ and $a_{1}^{\text {out }}, a_{2}^{\text {out }}$ are the input and output amplitudes of the optical power at the input and output ports, respectively. These can also be referred to as and correspond to input optical power at the input port 1 and 2 of the DC splitter and transmitted power through the parallel and cross port of the DC combiner.

The outpot power depends on effective refractive index of the waveguide, $n_{\text {eff }}$, difference in wavelength $(\Delta \lambda)$ between power output at port $1\left(P_{1}^{\text {Out }}(\lambda)\right)$ and port $2\left(P_{2}^{\text {Out }}(\lambda)\right)$, and difference in length $(\Delta \mathrm{L})$ between the straight and phase delay arms. In an ideal case, it can be given mathematically as, $P_{1}^{\text {out }}(\lambda)=\cos ^{2}\left(n_{\text {eff }} \pi \Delta \mathrm{L} / \lambda\right)$ and $P_{2}^{\text {Out }}(\lambda)=\sin ^{2}\left(n_{\mathrm{eff}} \pi \Delta \mathrm{L} / \lambda\right)$, where, the wavelength difference $(\Delta \lambda)$ between the output optical powers at port 1 and port 2 is given by: $\Delta \lambda=\frac{\lambda^{2}}{2 n_{\mathrm{eff}} \Delta \mathrm{L}}$.

The total transfer matrix of the MZI in turn can be represented as [5,44]: $\left(\begin{array}{l}a_{1}^{\text {out }} \\ a_{2}^{\text {out }}\end{array}\right)=\left(M_{S} M_{P S} M_{C}\right)\left(\begin{array}{c}a_{1}^{\text {in }} \\ a_{2}^{\text {in }}\end{array}\right)$, where, $M_{S}, M_{P S}$, and $M_{c}$ represent the amplitude transfer in the spliter, phase delay arm, and combiner, respectively. The transfer matrix for the phase shifter can be given by the product of transmission factor, $\gamma_{P S}=e^{-\alpha L_{P S}}$ and the phase factor $e^{-\beta L_{P S}}$ as: $M_{P S}=\gamma_{P S} e^{-j \beta L_{P S}}\left(\begin{array}{cc}e^{-j \alpha \Delta L} e^{-j \beta \Delta L} & 0 \\ 0 & 1\end{array}\right)$, where $\alpha$ is the optical loss and it is related to the absorption of radiation in the phase delay arm, LPS is the total length of the phase shifter arm, and $\beta$ is the wave propagation constant.

The sum of the transfer matrix can be obtained by combining the transfer matrix of the splitter phase-shifter, and combiner as: $M_{M Z I}=\gamma_{S} \gamma_{P S} e^{-j \beta . L_{P S}} \gamma_{\mathrm{c}}\left(\begin{array}{cc}p_{1} & j \cdot q_{1} \\ j \cdot q_{1} & p_{1}\end{array}\right)\left(\begin{array}{cc}e^{-j \alpha \Delta L} e^{-j \beta \Delta L} & 0 \\ 0 & 1\end{array}\right)\left(\begin{array}{cc}p_{2} & j \cdot q_{2} \\ j \cdot q_{2} & p_{2}\end{array}\right)$, where, the phase delay is given by: $\Delta \varphi=\beta \times \Delta L$. In the ideal case, when the input power gets divided between two ports in equal amount, the values of both the $\mathrm{p}$ and $\mathrm{q}$ become unity, which means that the power coming through the output port $1, P_{1}^{\text {out }}$, and output port $2, P_{2}^{\text {out }}$, of the device can be given as:

$$
\begin{aligned}
& P_{1}^{\text {out }}=\frac{1}{2} P_{1}^{\text {in }}\left(A_{1}-B \cos (\beta \Delta L)\right) \text { and } \\
& P_{2}^{\text {out }}=\frac{1}{2} P_{1}^{\text {in }}\left(A_{2}-B \operatorname{Sin}(\beta \Delta L)\right), \\
& A_{1}=2 \gamma_{S}^{2} \gamma_{P S}{ }^{2} \gamma_{c}^{2}\left(\kappa_{S} \kappa_{c}+\left(1-\kappa_{S}\right)\left(1-\kappa_{c}\right) e^{-2 \alpha \Delta L}\right), \\
& \text { here, } \quad A_{2}=2 \gamma_{S}^{2} \gamma_{P S}{ }^{2} \gamma_{c}^{2}\left(\kappa_{S}\left(1-\kappa_{c}\right)+\kappa_{c}\left(1-\kappa_{S}\right) e^{-2 \alpha \Delta L}\right), \text { and } \\
& \left.B=4 \gamma_{S}^{2} \gamma_{P S}{ }^{2} \gamma_{c}^{2} \sqrt{\left(\kappa_{S} \kappa_{c}\left(1-\kappa_{S}\right)\left(1-\kappa_{c}\right)\right.}\right) e^{-\alpha \Delta L} .
\end{aligned}
$$


Equation (1) defines three parameters, namely: Free spectral range (FSR), also referred to as separation between two adjacent / successive peaks of the transmission loss spectra; Insertion loss (IL), of the device, which is the absolute loss of transmission power in $\mathrm{dB}$ in each port of the MZI; and the separation between the adjacent maximum and minimum of the transmission spectra for each ports. For an ideal device IL would approach to $0 \mathrm{~dB}$ in each ports.
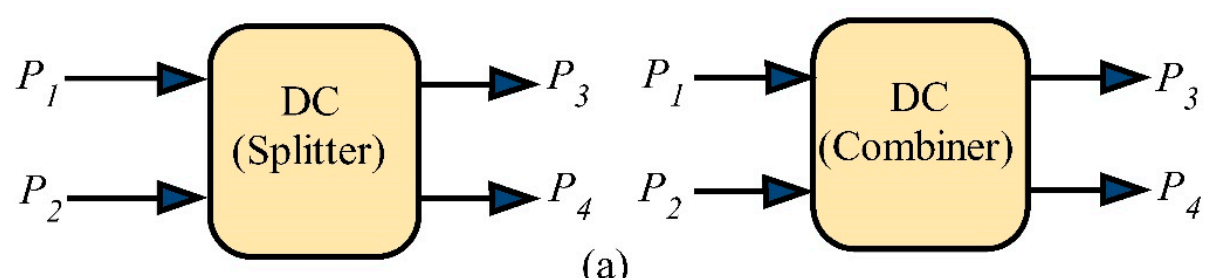

(a)

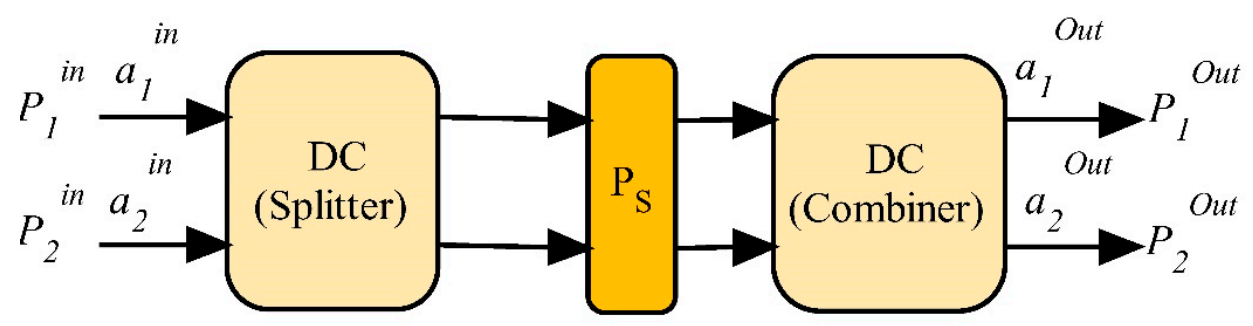

(b)

Figure 1 (a) Block diagram of directional couplers (DCs): DC power splitters and combiners. (b) Block diagram showing Mach-zehnder interferometer in cascade form of DC splitter, phase shifter arm (Ps), and DC combiner.

\section{Materials and Methods}

The Silicon used for the fabrication of the device was a material with a refractive index $\left(n_{S i}\right)$ of 3.48. The Silicon waveguide was sand-witched between two low-index claddings: air in the upper part and $\mathrm{SiO}_{2}$ of $99.99 \%$ purity in the lower part. A schematic of the Mach-zehnder interferometer is shown in Figure 2. The device consists of two 3-dB directional couplers (DC) - power combiners / splitters, and two MZI arms: straight arm of $3 \mathrm{~mm}$ length and a phase delay arm of $8.7 \mathrm{~mm}$ with serpentine architecture. The straight arm is separated from the nearest serpentine arm by $20.2 \mu \mathrm{m}$ gap. The phase delay arm consists of densely packed straight waveguides connected by C-shaped bends and sigmoidal connecter. These bends were introduced to reduce the coupling loss and to adjust the extinction ratio.

The radius of curvature of C-shaped bends and $S$-bend of the phase delay arms are kept at $3 \mu \mathrm{m}$ and $10 \mu \mathrm{m}$, respectively. Various waveguide design parameters are given in Table 1. Various geometrical parameters and optical properties of MZI, obtained from the simulation, are given in Table 2. 
Table 1: Geometrical parameters of optical waveguides.

\begin{tabular}{|l|l|l|l|}
\hline Parameters & Material & Thickness & Refractive index \\
\hline Upper cladding & Air & $450 \mathrm{~nm}$ & 1.00 \\
\hline Core & $\mathrm{Si}$ & $220 \mathrm{~nm}$ & 3.48 \\
\hline Lower cladding & $\mathrm{SiO}_{2}$ & $1000 \mathrm{~nm}$ & 1.45 \\
\hline
\end{tabular}

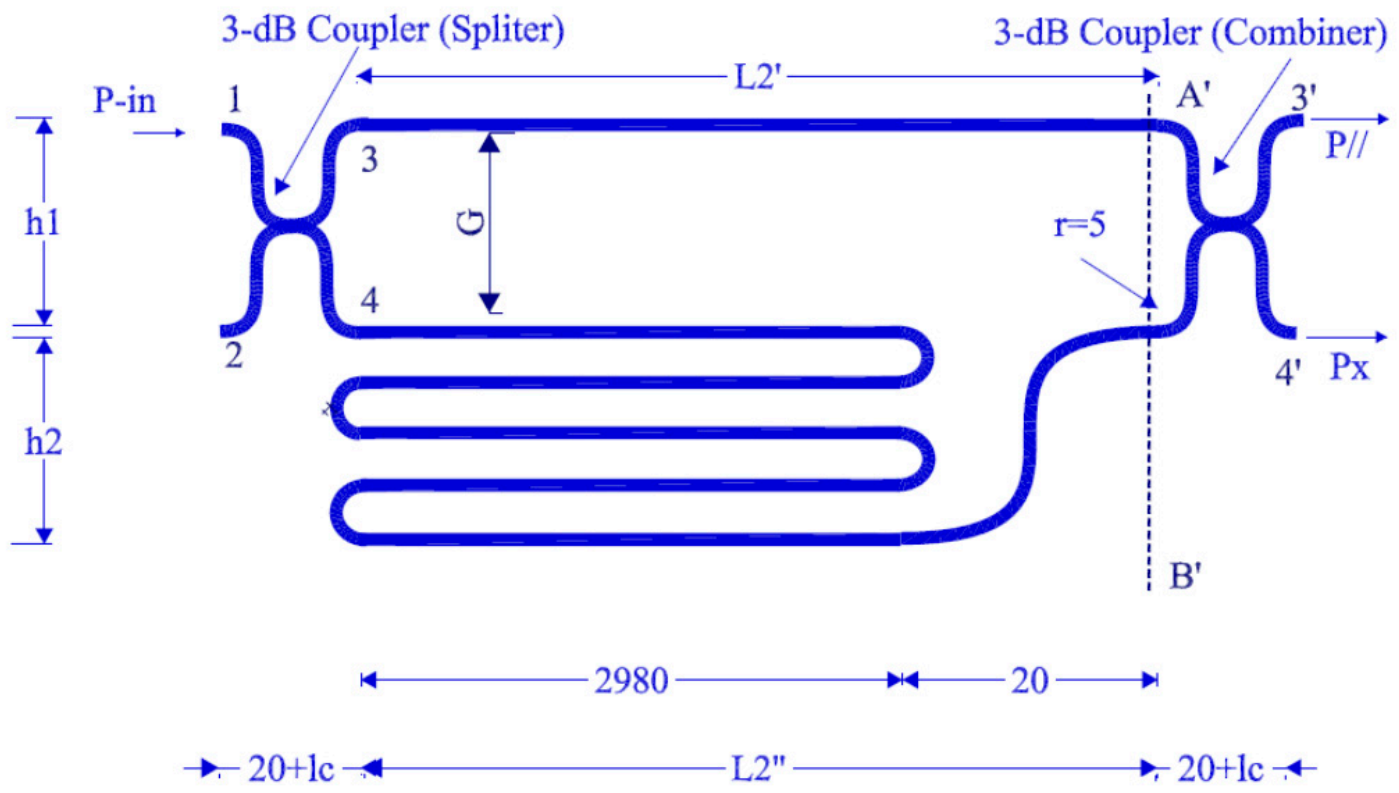

Figure 2 A schematic of the MZI with two symmetric directional couplers (DCs), straight and phase delay arms: The DC consists of a Si core surrounded by $\mathrm{SiO}_{2}$ cladding layer and air, coupling region with length of $24.5 \mu \mathrm{m}$ and separation between two inputs ( $\left.h_{1}\right)$ of $20.2 \mu \mathrm{m}$. The phase delay arm consists of C-shaped waveguides with radius of $3 \mu \mathrm{m}$ and S-shaped bends with radius of curvatures of $5 \mu \mathrm{m}$ and $10 \mu \mathrm{m}$. The refractive index $(n)$ of $\mathrm{Si}$ and $\mathrm{SiO}_{2}$ cladding are 3.48 and 1.45 , respectively. Cross section of the DC is denoted by $\mathrm{A}^{\prime} \mathrm{B}^{\prime}$.

Table 2: Geometrical and optical parameters at the wavelength $(\lambda)$ of $1550 \mathrm{~nm}$. Core height of $0.3 \mu \mathrm{m}$, Path difference between phase delay and straight arms of the MZI $(\Delta \mathrm{L})=5700 \mu \mathrm{m}, \mathrm{L}^{\prime}=3000 \mu \mathrm{m}, \mathrm{R}_{\mathrm{c}}=5 \mu \mathrm{m}, \mathrm{R}=$ $10 \mu \mathrm{m}, \mathrm{h}_{1}=20.2 \mu \mathrm{m}$. Waveguide parameters are denoted as follows: $\mathrm{w}=$ waveguide width, $\mathrm{g}=$ gap between parallel wavegudies, $\mathrm{dx}$ and $\mathrm{dy}=$ mesh sizes along $\mathrm{x}$ and $\mathrm{y}$ axis, $n_{e f f 1}$ and $n_{\text {eff } 2}=$ effective refractive indices of waveguide 1 and 2 of the $3-\mathrm{dB}$ couplers.

\begin{tabular}{c|c|c|c|c|c|c}
\hline \multicolumn{4}{c|}{ Geometrical Parameters } & \multicolumn{3}{c}{ Optical Parameters } \\
\hline $\mathrm{w}(\mathrm{nm})$ & $\mathrm{g}(\mathrm{nm})$ & $\mathrm{dx}(\mathrm{nm})$ & $\mathrm{dy}(\mathrm{nm})$ & $n_{\text {eff1 }}$ & neff2 & $3_{\mathrm{dB}} \mathrm{l}_{\mathrm{c}(\lambda)}(\mu \mathrm{m})$ \\
\hline 300 & 200 & 2.5 & 2.5 & 1.858 & 1.721 & 02.81 \\
\hline
\end{tabular}




\begin{tabular}{|c|c|c|c|c|c|c|}
\hline & & & 5.0 & 1.865 & 1.726 & 02.79 \\
\hline & & & 10.0 & 1.871 & 1.731 & 02.78 \\
\hline \multirow[t]{3}{*}{320} & 200 & 2.5 & 2.5 & 1.955 & 1.852 & 03.79 \\
\hline & & & 5.0 & 1.960 & 1.857 & 03.74 \\
\hline & & & 10.0 & 1.966 & 1.861 & 03.70 \\
\hline \multirow[t]{3}{*}{340} & 200 & 2.5 & 2.5 & 2.042 & 1.965 & 05.02 \\
\hline & & & 5.0 & 2.046 & 1.968 & 04.95 \\
\hline & & & 10.0 & 2.051 & 1.971 & 04.88 \\
\hline \multirow[t]{3}{*}{360} & 200 & 2.5 & 2.5 & 2.119 & 2.059 & 06.52 \\
\hline & & & 5.0 & 2.122 & 2.062 & 06.42 \\
\hline & & & 10.0 & 2.126 & 2.065 & 06.33 \\
\hline \multirow[t]{3}{*}{400} & 200 & 2.5 & 2.5 & 2.245 & 2.207 & 20.83 \\
\hline & & & 5.0 & 2.247 & 2.209 & 20.47 \\
\hline & & & 10.0 & 2.250 & 2.211 & 20.16 \\
\hline \multirow[t]{3}{*}{500} & 200 & 2.5 & 2.5 & 2.448 & 2.434 & 26.00 \\
\hline & & & 5.0 & 2.450 & 2.434 & 25.49 \\
\hline & & & 10.0 & 2.451 & 2.436 & 25.06 \\
\hline \multirow[t]{3}{*}{200} & 220 & 2.5 & 2.5 & 1.850 & 1.728 & 06.37 \\
\hline & & & 5.0 & 1.856 & 1.733 & 06.31 \\
\hline & & & 10.0 & 1.863 & 1.739 & 06.26 \\
\hline \multirow[t]{3}{*}{300} & 300 & 2.5 & 2.5 & 1.827 & 1.752 & 10.63 \\
\hline & & & 5.0 & 1.833 & 1.758 & 10.30 \\
\hline & & & 10.0 & 1.889 & 1.764 & 10.26 \\
\hline
\end{tabular}

Geometrical parameters of the device and its optical characteristics were optimized and validated using coupled mode theory discussed above and 2D / 3D vectorial mode solver.

\subsection{Mask Layout and Design Verification}

The mask layout for the device, including test structures, was prepared using DW-2000 design and verification tool, provided by CMC microsystems, Canada [45]. As shown in Figure 3, the main device part consists of a series of passive MZI and grating couplers. In this case both the waveguide height and width are fixed at $0.22 \mu \mathrm{m}$ and $0.30 \mu \mathrm{m}$, respectively to ensure single mode operation for TE polarized light at $\lambda=1550 \mathrm{~nm}$. To make sure that the device would be fabricated error free, foundry recommended design rules checking (DRC) were adopted with DW 2000 design tool that involved layout verification system (LVS). In a similar manner, the design of the mask layout was verified using LVS tool. This design tool was also employed to verify the following: 
a) Waveguide connectivity to minimize optical power losses;

b) Grids naming to make them in-line with version II of graphic database system (gds);

c) Alignment marking for subsequent lithography, and where applicable to define and verify coupling probing.

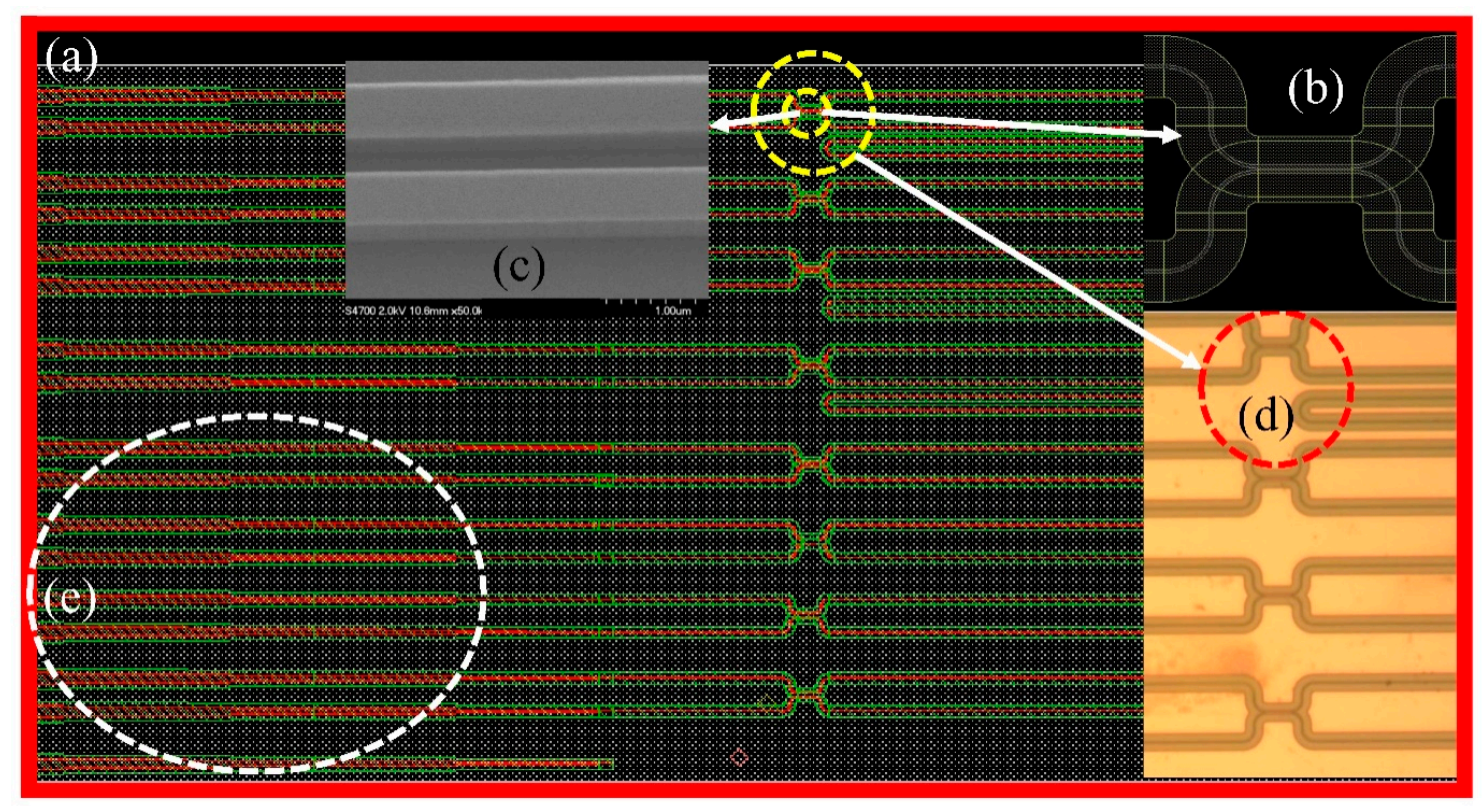

Figure 3 (a) Schematic diagram of the mask-layout designed using DW-2000, (b) enlarged view of 3dB couplers, (c) fabricated coupler waveguides (d) microscopic top view of the fabricated MZI device showing $3 \mathrm{~dB}$ coupler and both the straight and phase delay arms of MZI. (e) mask-layout of the vertical grating coupler, encircled by white dash lines.

\subsection{Simulation Set-up}

The grid sizes in the range of $2.5 \mathrm{~nm}$ to $10.0 \mathrm{~nm}$ were used during FDTD simulation for a waveguide dimension of $0.3 \mu \mathrm{m}$ width and $0.22 \mu \mathrm{m}$ thickness, and a gap of $0.2 \mu \mathrm{m}$ between parallel waveguides. To minimize error, two mesh override regions were used. One region covered both the waveguides and the region outside them that extend to $100 \mathrm{~nm}$ to account for evanescent fields. Since the coupling of guided modes are very sensitive to the gap between the waveguides, a second region was set with a mesh grid size of $2 \mathrm{~nm}$, which ensured more accurate resolving of optical modes both inside waveguides and in evanescent.

\subsection{Fabrication Specifications}

The device was fabricated at IMEC in Belgium, with different device dimension using a UV lithography of 193-nm wavelength on a SOI platform $[5,40,46]$. Two types of mask levels were employed: $0.220 \mu \mathrm{m}$ deep etch for the waveguides and $0.070 \mu \mathrm{m}$ shallow etch for vertical grating 
couplers. Sidewall roughness was controlled to approximately $0.005 \mu \mathrm{m}$ through hardening plasma treatment.

\subsection{Test Set-Up And Measurement Schematics}

Far-field measurement setup was used to measure the optical characteristics of the fabricated device. A schematic of the experimental set-up for the measurement of transmission loss and FSR is displayed in Figure 4. As shown, it consisted of a tunable laser (Agilent 8164A) with wavelength range of $1510 \mathrm{~nm}$ - $1640 \mathrm{~nm}$, TE polarizer, charge coupled camera, grating coupler, MZI, heating arrangement, and a photo detector (Thorlabs PDA30G). Vertical fiber couplers have been used at the input and output for optical excitation and collection.

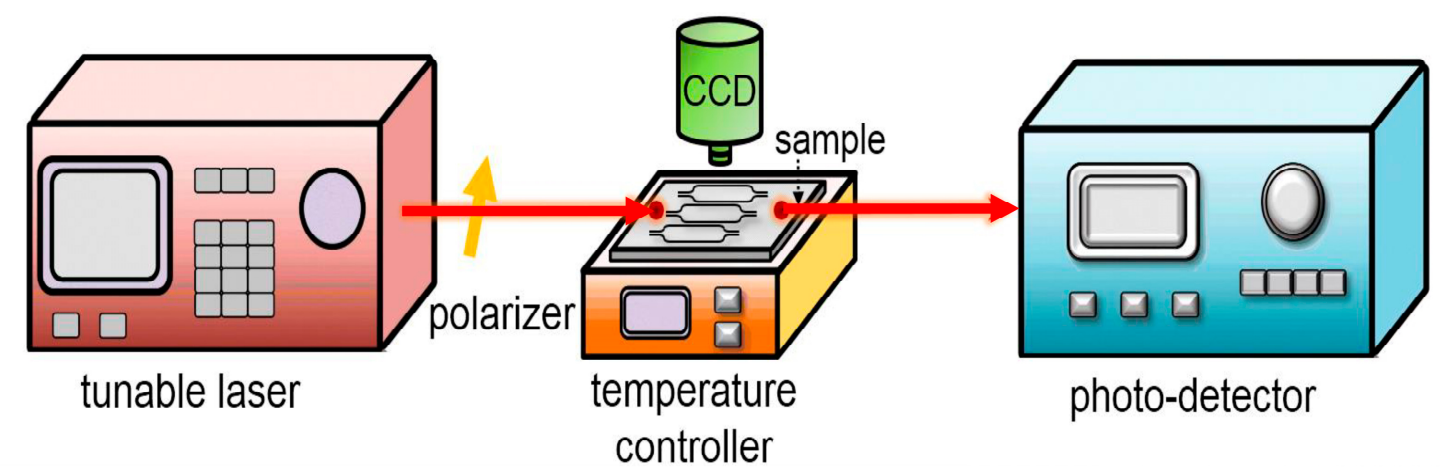

Figure 4: Schematic of an experimental set-up for transmission loss / spectra measurement

This laser supports a single-mode lensed fiber and broad band polarizer to ensure TE polarized mode. As shown in this Figure, a laser signal was fed to the grating coupler using a lensed fiber. A TE polarizer was placed in between the laser source and the lensed fiber so as to TE polarize the input signal. Since the transmission loss characteristics of MZI based interferometer is strongly depended on optical polarization, only the output power for TE polarization mode is measured. The tapper grating has period of $620 \mathrm{~nm}, 40-\mathrm{nm}$ per 1-dB bandwidth, 5.1-dB coupling losses at $1550 \mathrm{~nm}$, $20 \mu \mathrm{m}$ by $10 \mu \mathrm{m}$ area, and 10-degree vertical angle tolerance [5,47].

A Charged-coupled Device (CCD) camera was used to assist positioning of both the input power feeder and output power collector fibers to and from the device, respectively. To ease the alignment of the lensed fiber with the grating coupler a lamp and a microscope camera were also utilized. The optical radiation was coupled through the input grating coupler and the signal was extracted through the output coupler grating and lensed fiber. The transmitted power was measured using an optical detector (Agilent 81525A and 81533B). The position of the lensed fiber was controlled by piezoelectric controller that allowed scanning every input and output radiation signal. 
The FSR was obtained from the transmission loss spectrum. The temperature of the MZI device was controlled using a tunable temperature controller.

\section{Results and Discussions}

The wavelength $(\lambda)$ dependent optical field profiles and the coupler and phase shifter arm's length dependent power characteristics were obtained using 2D / 3D-FDTD simulation tool. Figure 5 shows the optical mode profiles of the waveguide at various widths - ranging from $0.3 \mu \mathrm{m}$ to 0.5 $\mu \mathrm{m}$. As identified by the field profiles shown in Figure 5 (a) through (f), these waveguides support single mode at $\lambda=1550 \mathrm{~nm}$. Figure $5(\mathrm{~g})$ shows an effective refractive index $\left(n_{\text {eff }}\right)$ of the waveguide as a function of width. As shown in it, the $n_{\text {eff }}$ increases almost linearly with the width of the waveguide.

Figure 5: Simulated mode profiles of Si based waveguide with width (a) $0.5 \mu \mathrm{m}$, (b) $0.4 \mu \mathrm{m}$, (c) $0.36 \mu \mathrm{m}$, (d) $0.34 \mu \mathrm{m}$, (e) $0.32 \mu \mathrm{m}$, and (f) $0.30 \mu \mathrm{m}$. Mesh size of $5 \mathrm{~nm}$ was used in the simulation. (g) Effective refractive index $\left(n_{e f f}\right)$ as a function of the waveguide width obtained from (a-f).

Optical field profiles of the straight arms of the test DC device obtained using the Lumerical mode solver functionality of FDTD simulation tool is given in Figure 6. In this case the optical radiation was fed to input ports $\mathrm{P}_{1}$ and $\mathrm{P}_{2}$ of the MZI (see, Figure 2). The device tested consisted of a fixed gap $(\mathrm{g})$ of $0.2 \mu \mathrm{m}$ and the width $(\mathrm{w})$ of $0.3 \mu \mathrm{m}$ with an input light signal launched at $\lambda=1550$ nm.

The 3-dB coupling lengths (3-дв $l_{c(\lambda)}$ ) of the DC was found to be highly dependent on the mesh size used during simulation. These are shown in Table 2 . The 3-дв $l_{c(\lambda)}$ ranged from 2.81 to 26 for a waveguide width ranging from 0.3 to $0.5 \mu \mathrm{m}$ and for a device with a constant $\mathrm{g}$ of $0.2 \mu \mathrm{m}$, indicating 
that these miniature coupled waveguides show a strong optical coupling effect. The $3-\mathrm{dB} l_{c(\lambda)}$ is remarkably sensitive to the changes in the $n_{\text {eff }}$ of the material. As shown in Table 2, the $n_{\text {eff }}$ of the waveguide changes significantly with changes in both $\mathrm{w}$ and $\mathrm{g}$ from $0.3 \mu \mathrm{m}$ to $0.5 \mu \mathrm{m}$ and $0.2 \mu \mathrm{m}$ to $0.3 \mu \mathrm{m}$, respectively.
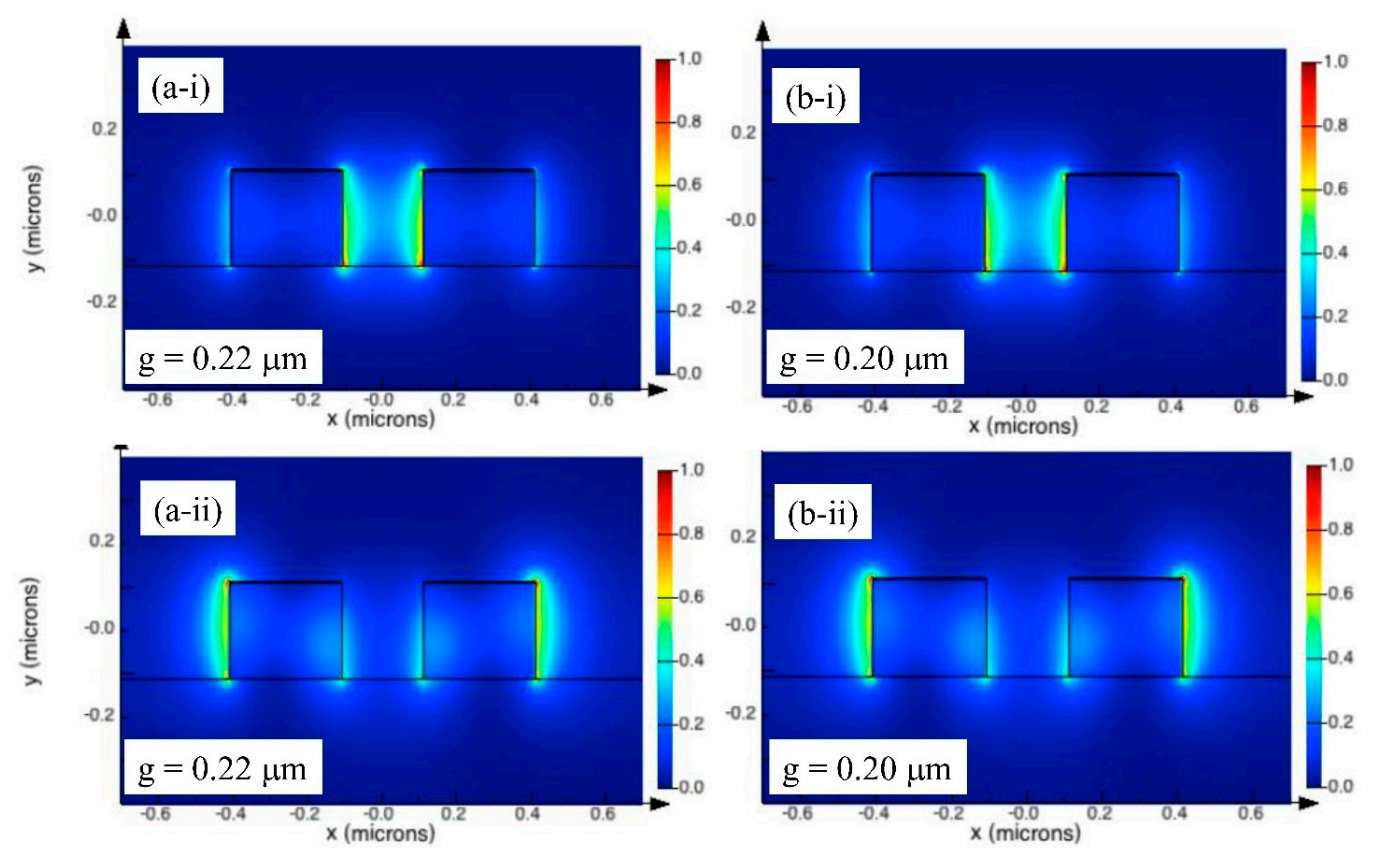

Figure 6: Mode profiles of parallel waveguides of direction couplers (a-i, b-i) for symmetric (a-ii, b-ii) for asymmetric modes. Cross-sectional area of both the waveguides is $0.3 \times 0.22 \mu \mathrm{m}^{2}$, coupling gap (g) between the parallel waveguides for (a) is $0.22 \mu \mathrm{m}$ and for (b) is $0.20 \mu \mathrm{m}$. Symmetric mode effective refractive index, $n_{e f f(a-i)}=1.56$ and asymmetric mode refractive index, $n_{e f f(a-i i)}=1.39$, (b) $\mathrm{g}$ of 0.20 , symmetric mode, $n_{\text {eff }(b-i)}=1.571$ and asymmetric mode, $n_{e f f(b-i i)}=1.378$.

Figure 7 (a) demonstrates changes in coupling length, $l_{c(\lambda)}$ as a function of waveguide width, as the gap between them is changed from $0.05 \mu \mathrm{m}$ to $0.3 \mu \mathrm{m}$. As displayed in this Figure, $l_{c(\lambda)}$ increases monotonously with increasing both the width and gap of the coupled waveguides.

Electric field profiles of both the symmetric and anti-symmetric super-modes are shown in Figure 7(b) with the separation of $0.2 \mu \mathrm{m}$ between the coupled waveguides. Figure 7(c) displays how the effective refractive index of the waveguide changes with the wavelength of the incident light / input light signal: The effective refractive index of the waveguide changes significantly with the $\lambda$ of the incident optical radiation.

Coupling length $\left(l_{c}\right)$ of the two parallel waveguides with a cross-sectional area of $0.3 \times 0.22 \mu \mathrm{m}^{2}$ for each was calculated using the index difference between the symmetric and anti-symmetric modes, obtained from both the Lumerical mode solver and FDTD simulation tools. Assuming 
coupling of $50 \%$ of power from waveguide 1 (in this case of the parallel port, $\mathrm{P} / /$ ) to waveguide 2 (cross port, $\mathrm{P} \perp$ ), the $3-\mathrm{dB}$ power coupling length is estimated to be $2.28 \mu \mathrm{m}$.

Figure 7(d) shows the calculated out-put power of the DC coupler for the coupled waveguide length that ranged from 0 to $5 \mu \mathrm{m}$. As shown in this figure, the output power from DC are complementary and change sinusoidally with the length of the waveguide.

Figure 7: (a) Waveguide width and gap dependence profile of coupling length. (b-i) Schematics of waveguides separated by $0.2 \mu \mathrm{m}$ gap. (b-ii) Electric field profiles for symmetric and anti-symmetric super-modes. Blue loops represent identical field value of $3 \mathrm{~dB}$ as represented by step between lines showing fundamental electric field distribution and E-field intensity: Wavelength $(\lambda)=1550 \mathrm{~nm}$, coupler gap $(\mathrm{g})=0.2 \mu \mathrm{m}$, and waveguide cross-section $=0.66 \mu \mathrm{m}^{2}$. (c) Diagram showing the change in effective refractive index of the waveguide with respect to the $\lambda$ of the incident optical radiation. (d) Plot of calculated output power versus position of the straight waveguide of the parallel $(/ /)$ and cross $(\perp)$ ports of the directional coupler calculated using (b) and (c) at $\lambda=1550 \mathrm{~nm}$. Red dots denote power in the // and blue circles denote power in the $\perp$ ports.

Figure 8 (a) shows transmitted power loss spectra of an ideal MZI device with $\gamma_{M Z I}=1.0$ and in the wavelength $(\lambda)$ range of $1549.5 \mathrm{~nm}-1551.5 \mathrm{~nm}$ as obtained from simulation. In the given $\lambda$ range, it showed 4 transmission loss bands / peaks with free-spectral range (FSR) equal to $0.26 \mathrm{~nm}$ (FSR is the spectral period of the transmission curve). As shown in this Figure, the output powers were found to be complementary, and they changed sinusoidally as the coupling wavelength is changed. This indicates that the device can be used as a wavelength selective high speed optical switch. The 
insertion loss, which is estimated from the absolute maximum value of the transmission bands in $\mathrm{dB}$ for each port, is $0 \mathrm{~dB}$ in both ports.

Ideally, the isolation of the MZI device, which is the separation between maximal and minimal magnitude of the transmission loss spectrum, is infinite. Note that the power loss exhibited by the device in (a) in no circumstances account for the loss due to Sigmoidal and C-bends and the loss associated with the contact between input and output fiber to the grating coupler.

Figure 8(a) Wavelength dependent simulated transmission loss characteristics of the Mach-zehnder interferometer at room temperature with optical absorption coefficient $(\alpha)$ of $0 \mathrm{~dB}$ and with both the coupling coefficients, $k_{S}=k_{c}$ each equal to $1 / 2$ and the transmission coefficient $\gamma_{M Z I}$ equal to 1 . The isolation between two ports is infinite $(\infty)$ and the free spectral range (FSR) is $0.26 \mathrm{~nm}$. (b) Transmission loss characteristics of the asymmetrical MZI with $\alpha=-0.45 \mathrm{~dB}, k_{s}=k_{c}$ each equal to $1 / 2$, and the 
transmission coefficient $\gamma_{M Z I}$ equal to 0.75. (c) Wavelength dependent experimental and fitted transmission loss characteristics measured at room temperature.

For a non-ideal case with a phase delay arm loss of $0.45 \mathrm{~dB}$ as identified by $\gamma_{M Z I}=0.75$, the transmitted power loss spectra of the device in the wavelength range of $1549.5 \mathrm{~nm}-1551.5 \mathrm{~nm}$ is given in Figure 8(b). In the given $\lambda$ range, it again showed 4 transmission loss peaks with the FSR equal to $0.26 \mathrm{~nm}$. Note that the insertion loss is non-zero in both ports. The isolation of the device is non-infinite in this case as identified by minimal transmission of below zero $\mathrm{dBm}$. As depicted in the figure, a slight variation in transmission coefficient and in the presence of a loss in the phase delay arm, optical interference is highly affected, leading to a strong optical isolation between the output ports 1 and 2 . The coupling associated with the S-shaped waveguides was estimated by extrapolation and these corresponded to a coupling length of $2 \mu \mathrm{m}$, similarly to those reported by Yamada et al. [48,49].

Figure 8 (c) shows the measured wavelength dependent transmission loss characteristics of the MZI device for the $\mathrm{P}_{/ /}$case. In the given $\lambda$ range, it again showed 4 transmission loss peaks with the FSR equal to $0.245 \mathrm{~nm}$. The insertion loss is approximately $25 \mathrm{dBm}$ and it increases with increasing wavelength. The discrepancies between the simulated (a-b) and measured (c) transmission loss spectra have been large and these differences can be attributed to several sources including assumption of negligible bending losses during modeling, larger surface roughness, imperfections, and defects in fabricated device, and contact losses between input and output fiber to the grating coupler during measurements.

Figure 9(a) Measured wavelength dependent transmission loss characteristics of the MZI at $\mathrm{T}=24^{\circ} \mathrm{C}($ dark blue), $25{ }^{\circ} \mathrm{C}$ (dark yellow), $26{ }^{\circ} \mathrm{C}$ (dark green), and $27{ }^{\circ} \mathrm{C}$ (dark red). A large light blue arrow shows the direction of shift of transmission loss peak with increasing substrate temperature: The changes displayed here are in reference to the peak positions at $24{ }^{\circ} \mathrm{C}$. The notations $\mathrm{P}_{1}, \mathrm{P}_{2}, \mathrm{P}_{3}$ and $\mathrm{P}_{4}$ denote four different peak positions between the $1449.5 \mathrm{~nm}$ and $1550 \mathrm{~nm}$ wavelength range. (b) Effect of temperature on the transmission loss spectra at the given wavelength range deduced from (a). 
Temperature dependent transmission loss spectra obtained from the experiment are depicted in Fig. 9 (a). The results suggest temperature change resulting to profound effects on both the peak positions and peak amplitude of the transmission loss spectra. These transmission loss spectra were measured with vertical optical fibers that were coupled to both the input and output ports of the MZI device. The method employed is similar to the one described by Yamada et al. [50]. For the given wavelength range, the general trend is that the peak positions are shifted to the longer wavelength with increasing temperature from $24^{\circ} \mathrm{C}$ to $27^{\circ} \mathrm{C}$.

Figure 9 (b) shows the temperature dependent wavelength shift recorded from figure 9(a) for four different peak positions, $\mathrm{P}_{1}$ through $\mathrm{P}_{4}$. As shown in the figure, the peak positions monotonously increase at a rate of approximately $0.1 \mathrm{~nm} /{ }^{\circ} \mathrm{C}$. The measured results clearly show the highly sensitive temperature dependent shift of transmission loss spectra and the reasonably high extinction ratio. These characteristics are greatly favorable for development of highly sensitive nanoscale thermal sensors.

\section{Conclusions}

A MZI based thermo-optical switch was designed, fabricated and characterized. It consisted of $3 \mathrm{~dB}$ couplers with straight and phase delay arms. The waveguide was fabricated onto Si waveguides using SOI technology. Its performance characteristics were evaluated using transmission loss and temperature dependent phase shift measurements. The coupling strength of the DC is found to be remarkably sensitive to the gap between the parallel waveguides. The fabricated DC coupler showed extremely short coupling length, typically ranging from $2 \mu \mathrm{m}$ to $10 \mu \mathrm{m}$. It means that in addition to being useful as thermal sensor, these devices can be used as compact power dividers or combiners and optical switches at optical communication wavelength. The transmission loss spectra of the MZI showed a strong sensitivity to the temperature changes, meaning that the device can be used as a highly sensitive thermal sensor, among many others.

Acknowledgments: C. Rizal thanks Drs. Nicolas A. F. Jaeger and Lukas Chrostowski at the University of British Columbia and Dan Daptic at the CMC Microsystems, Canada for their feedback and critical comments during planning and execution of this work.

Author Contributions: C.R. conceived and designed the experiments; C.R and B.N. performed the experiments; C.R. and B.N. analyzed the data; C. R. wrote the paper and all the authors contributed equally.

Conflicts of Interest: The authors declare no conflict of interest.

The following abbreviations are used in this manuscript: 
MZI Mach-zehnder Interferometer

3D there-dimensional

2D two-dimensional

DC directional coupler

FDTD finite-difference time domain

Matlab matrix laboratory

\section{References}

1. Jalali, B.; Yegnanarayanan, S.; Yoon, T.; Yoshimoto, T.; Rendina, I.; Coppinger, F. Advances in silicon-on-insulator optoelectronics. IEEE Journal of selected topics in Quantum Electronics 1998, 4, 938-947.

2. Lipson, M. Guiding, modulating, and emitting light on silicon-challenges and opportunities. Journal of Lightwave Technology 2005, 23, 4222-4238.

3. Kimerling, L.C. Silicon microphotonics. Applied Surface Science 2000, 159, 8-13.

4. Boeck, R.; Caverley, M.; Chrostowski, L.; Jaeger, N.A.F. Experimental demonstration of a silicon-on-insulator high-performance double microring filter using mzi-based coupling. Optics Letters 2015, 40, 276-279.

5. Chrostowski, L.; Hochberg, M. Silicon photonics design: From devices to systems. Cambridge University Press: 2015.

6. Chrostowski, L.; Wang, X.; Flueckiger, J.; Wu, Y.; Wang, Y.; Fard, S.T. In Impact of fabrication non-uniformity on chip-scale silicon photonic integrated circuits, Optical Fiber Communication Conference, 2014, Optical Society of America: p Th2A. 37.

7. Xu, K.; Ning, N.; Ogudo, K.A.; Polleux, J.-L.; Yu, Q.; Snyman, L.W. In Light emission in silicon: From device physics to applications, International Workshop on Thin Films for Electronics, Electro-Optics, Energy and Sensors, 2015, International Society for Optics and Photonics: pp 966702-966702-966710.

8. Pavesi, L. Will silicon be the photonic material of the third millenium? Journal of Physics: Condensed Matter 2003, 15, R1169.

9. Pavesi, L.; Lockwood, D.J. Silicon photonics. Springer Science \& Business Media: 2004; Vol. 1.

10. Graydon, O. View from... Group iv 2015: The data centre challenge. Nature Photonics 2015, 9, 637-638.

11. Pavesi, L.; Lockwood, D.J. Silicon photonics iii. 2016.

12. Liu, Q.; Tu, X.; Kim, K.W.; Kee, J.S.; Shin, Y.; Han, K.; Yoon, Y.-J.; Lo, G.-Q.; Park, M.K. Highly sensitive mach-zehnder interferometer biosensor based on silicon nitride slot waveguide. Sensors and Actuators B: Chemical 2013, 188, 681-688. 
13. Yuan, D.; Dong, Y.; Liu, Y.; Li, T. Mach-zehnder interferometer biochemical sensor based on silicon-on-insulator rib waveguide with large cross section. Sensors 2015, 15, 21500-21517.

14. Vivien, L.; Pavesi, L. Handbook of silicon photonics. Taylor \& Francis: 2013.

15. Pavesi, L.; Gaponenko, S.V.; Dal Negro, L.; NATO Advanced Research Workshop on towards the first silicon laser : :. Towards the first silicon laser. Kluwer Academic Publishers: Dordrecht, 2003; p 482 s.

16. Jalali, B.; Yegnanarayanan, S.; Yoon, T.; Yoshimoto, T.; Rendina, I.; Coppinger, F. Advances in silicon-on-insulator optoelectronics. Selected Topics in Quantum Electronics, IEEE Journal of 1998, 4, 938-947.

17. Bogaerts, W.; Fiers, M.; Dumon, P. Design challenges in silicon photonics. Selected Topics in Quantum Electronics, IEEE Journal of 2014, 20, 1-8.

18. Bauters, J.F.; Davenport, M.L.; Heck, M.J.R.; Doylend, J.K.; Chen, A.; Fang, A.W.; Bowers, J.E. Silicon on ultra-low-loss waveguide photonic integration platform. Optics express 2013, 21, 544-555.

19. Gautam, R. Silicon microring resonator loaded mach-zehnder interferometer for low power optical modulation, switching and bio-sensing. Yokohama National University, Japan, 2014.

20. Hafezi, M.; Mittal, S.; Fan, J.; Migdall, A.; Taylor, J.M. Imaging topological edge states in silicon photonics. Nature Photonics 2013, 7, 1001-1005.

21. Koos, C.; Freude, W.; Leuthold, J.; Kohl, M.; Dalton, L.R.; Bogaerts, W.; Lauermann, M.; Melikyan, A.; Wolf, S.; Weimann, C. In Silicon-organic (soh) and plasmonic-organic (poh) hybrid integration: Extending the capabilities of silicon photonics and plasmonics, Opto-Electronics and Communications Conference (OECC), Shanghai, China, 2015, IEEE Photonics: Shanghai, China, pp 1-2.

22. Li, Q.; Davanco, M.; Srinivasan, K. Efficient and low-noise single-photon-level frequency conversion interfaces using silicon nanophotonics. arXiv preprint arXiv:1510.02527 2016.

23. Madsen, C.K.; Zhao, J.H. Optical filter design and analysis: A signal processing approach john wiley \& sons. Inc.US, New York 1999.

24. Najari, M.; Javan, A.M.; Amiri, N. Hybrid all-fiber sensor for simultaneous strain and temperature measurements based on mach-zehnder interferometer. Optik-International Journal for Light and Electron Optics 2015, 126, 2022-2025.

25. Novak, J. Design, fabrication, and packaging of mach-zehnder interferometers for biological sensing applications. 2015.

26. Pacholski, C. Photonic crystal sensors based on porous silicon. Sensors 2013, 13, 4694-4713. 
27. Pond, J.; Cone, C.; Chrostowski, L.; Klein, J.; Flueckiger, J.; Liu, A.; McGuire, D.; Wang, X. In A complete design flow for silicon photonics, SPIE Photonics Europe, 2014, International Society for Optics and Photonics: pp 913310-913310-913317.

28. Priolo, F.; Gregorkiewicz, T.; Galli, M.; Krauss, T.F. Silicon nanostructures for photonics and photovoltaics. Nature nanotechnology 2014, 9, 19-32.

29. Shoji, Y.; Shirato, Y.; Mizumoto, T. Silicon machâ€“"zehnder interferometer optical isolator having $8 \mathrm{~nm}$ bandwidth for over $20 \mathrm{db}$ isolation. Japanese Journal of Applied Physics 2014, 53, 022202 .

30. Ni, X.; Wang, M.; Guo, D.; Hao, H.; Zhu, J. A hybrid mach-zehnder interferometer for refractive index and temperature measurement. IEEE Photonics Technology Letters 2016, 1-6.

31. Barrios, C.A.; De Almeida, V.R.; Lipson, M. Low-power-consumption short-length and high-modulation-depth silicon electrooptic modulator. Lightwave Technology, Journal of 2003, 21, 1089-1098.

32. Yariv, A.; Yeh, P. Photonics: Optical electronics in modern communications (the oxford series in electrical and computer engineering). Oxford University Press, Inc.: 2006.

33. Tang, T.; Luo, L.; Liu, W.; He, X.; Zhang, Y. Thermo-optic goos-hanchen effect in silicon-on-insulator waveguide. Applied Physics B 2015, 120, 497-504.

34. Soref, R.A.; Bennett, B.R. Electrooptical effects in silicon. Quantum Electronics, IEEE Journal of 1987, 23, 123-129.

35. Dong, P.; Duan, G.-H.; Chen, Y.-K.; Neilson, D.T. Merits and potential impact of silicon photonics. In Silicon photonics iii, Springer: 2016; pp 397-420.

36. Pokharel, R.K.; Rizal, C.S. In Design and characterization of a compact optical device based on mach-zehnder interferometer in silicon-on-insulator technology, 15th Annual Meetting of the Pacific Center for Advanced Materials (PCAMM), Vancouver, Canada, August 15, 2011, 2011; Vancouver, Canada.

37. Takahashi, Y.; Inui, Y.; Chihara, M.; Asano, T.; Terawaki, R.; Noda, S. A micrometre-scale raman silicon laser with a microwatt threshold. Nature 2013, 498, 470-474.

38. Okamoto, K. Progress and technical challenge for planar waveguide devices: Silica and silicon waveguides. Laser \& photonics reviews 2012, 6, 14-23.

39. Kawachi, M. Silica waveguides on silicon and their application to integrated-optic components. Optical and Quantum Electronics 1990, 22, 391-416.

40. Bogaerts, W.; Selvaraja, S.; Dumon, P. Technology paper imec. ePIXfab, Gent, Belgium 2008. 
41. Soref, R. The past, present, and future of silicon photonics. IEEE Journal of selected topics in quantum electronics 2006, 12, 1678-1687.

42. Khriachtchev, L. Silicon nanophotonics: Basic principles, current status and perspectives. Pan Stanford Publishing: 2009.

43. Doylend, J.K.; Knights, A.P. The evolution of silicon photonics as an enabling technology for optical interconnection. Laser $\mathcal{E}$ Photonics Reviews 2012, 6, 504-525.

44. Ducournau, G.; Latry, O.; Ketata, M. The all-fiber mzi structure for optical dpsk demodulation and optical psbt encoding. Journal of Systemics, Cybernetics and Informatics 2006, 4, 78-89.

45. CMC Microsystem. Nano silicon-on-isulator process technology. http://www.cmc.ca/

46. IMEC ePIXfab. European alliance of organizations. http://www.epixfab.eu/index.php

47. Xia, F.; Sekaric, L.; Vlasov, Y.A. Mode conversion losses in silicon-on-insulator photonic wire based racetrack resonators. Optics express 2006, 14, 3872-3886.

48. Yamada, H.; Tao, C.H.U.; Ishida, S.; Arakawa, Y. Si photonic wire waveguide devices. IEICE Transactions on Electronics 2007, 90, 59-64.

49. Kobayashi, N.; Sato, K.; Namiwaka, M.; Yamamoto, K.; Watanabe, S.; Kita, T.; Yamada, H.; Yamazaki, H. Silicon photonic hybrid ring-filter external cavity wavelength tunable lasers. Journal of Lightwave Technology 2015, 33, 1241-1246.

50. Yamada, K.; Tsuchizawa, T.; Nishi, H.; Kou, R.; Hiraki, T.; Takeda, K.; Fukuda, H.; Ishikawa, Y.; Wada, K.; Yamamoto, T. High-performance silicon photonics technology for telecommunications applications. Science and Technology of Advanced Materials 2016.

(C) 2016 by the authors; licensee Preprints, Basel, Switzerland. This article is an open access article distributed under the terms and conditions of the Creative Commons by Attribution (CC-BY) license (http://creativecommons.org/licenses/by/4.0/). 